\title{
Front-face and right-angle fluorescence spectroscopy for monitoring extra virgin olive oil spectrum evolution
}

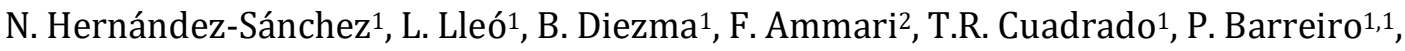 \\ J.M. Roger ${ }^{2}$ and M. Ruiz-Altisent ${ }^{1}$ \\ 1Physical Properties Laboratory and Advanced Technologies in Agrifood, LPF-TAGRALIA, ETSIAAB, Universidad \\ Politécnica de Madrid, Madrid, Spain; ${ }^{2}$ Irstea, UMR ITAP, 361 Rue J.F. Breton, Montpellier Cedex 5, France.
}

\begin{abstract}
Fluorescence spectroscopic techniques were applied in a study in order to monitor the evolution of the spectral pattern of extra virgin olive oil (EVO0) samples exposed to indirect light at room temperature. Detailed information was extracted from three-dimensional front-face (FF) fluorescence spectra with excitation wavelengths ranging from 230 to $646 \mathrm{~nm}$ and emission wavelengths ranging from 250 to $698.5 \mathrm{~nm}$. Relevant emission regions were revealed in FF experiments that were useful to study the variability of the characteristic spectral patterns and to develop fast inspection procedures. Such procedures include simultaneous excitation at wavelengths lower than $400 \mathrm{~nm}$, which was proposed and implemented in a rightangle prototype in order to monitor the evolution of EVOO samples exposed to indirect light. Emission signals at local maxima around 400, 434, 464, 513 and $674 \mathrm{~nm}$ were found to be relevant. Ongoing research highlights that hyperspectral images provide spectral patterns of the olives, allowing more precise sorting into categories, which would enable the classification into lots of oils with more homogeneous characteristics for subsequent modelling.
\end{abstract}

Keywords: oil quality, oil storage, oxidation, principal component analysis

\section{INTRODUCTION}

Olive oil consumption is spreading all over the world, promoted by the sensorial attributes and health benefits associated with the chemical and nutritional composition of extra virgin olive oil (EVOO) (López-Miranda et al., 2010). Under oxidation-inducing conditions promoted by illumination, a sample of olive oil might contain a plethora of oxidation compounds. Most of the olive oil components involved in the oxidation processes, such as chlorophyll, antioxidant compounds (polyphenols and $\alpha$-tocopherol) and primary and secondary oxidation products, are fluorescent molecules (Sikorska et al., 2012). Thus, a complex fluorescence spectral pattern is expected to reveal the oxidation status. Fast inspection procedures are of great interest. Hence, identification of the bands of excitation wavelengths that provide information on oxidation evolution is a major issue to develop a fast procedure with simultaneous excitation that gives rise to useful emission signals. The present work shows the preliminary results of a study where detailed regions of excitationemission wavelengths have been extracted from three-dimensional front-face (FF) fluorescence spectra. Simultaneous excitation at the wavelengths of interest has been proposed and implemented in a right-angle (RA) prototype in order to monitor the evolution of EVOO samples exposed to indirect light.

\footnotetext{
${ }^{1}$ E-mail: pilar.barreiro@upm.es
} 
The hyperspectral imaging system allows integrating spectroscopic and imaging techniques to enable direct identification of different components or quality characteristics and their spatial distribution within the tested samples (Lara et al., 2013; Lleó et al., 2011). Hyperspectral imaging techniques have emerged as a powerful tool in agricultural and food systems, as they overcome the limitations of spectroscopic and vision techniques.

Hyperspectral images could provide detailed spectral patterns useful to characterize olives and to sort them into homogeneous categories before oil extraction.

\section{MATERIALS AND METHODS}

Set 1 included two EVOO samples obtained directly from producers (P1 and P2) and five EVOO samples (according to commercial labelling) acquired from retail markets (C1, C2, C3, C6 and C8), and seven olive oil samples from retail markets (001-007) (Table 1). 3D FF and RA fluorescence spectra were obtained from each sample, and then analysed and compared in order to assess the most suitable configuration of the RA experiments. Set 2 included 13 EVOO samples, according to commercial labelling, obtained from retail markets. Commercial samples (C1-C13) included monovarietal oils, known varietal blends and unknown varietal blends (Table 2). These samples were exposed to indirect light in transparent glass bottles at room temperature for 5 months. RA fluorescence spectra were acquired monthly from each sample from the control month at the beginning of the experiment to the fifth month under treatment. Principal component analysis (PCA) was performed on centred data. Set 3 included olive fruit samples, cultivar 'Picual', sorted visually into group 1 (G1, green and 'pintonas' olives), G2 (purple olives) and G3 (black olives).

Table 1. Characteristics of extra virgin olive oil (EVOO) and olive oil (00) samples of set 1 analysed by 3D front-face and right-angle fluorescence spectroscopy.

\begin{tabular}{|c|c|c|c|}
\hline \multirow{2}{*}{\multicolumn{4}{|c|}{$\begin{array}{l}\text { Sample } \\
\text { EVOO }\end{array}$}} \\
\hline & & & \\
\hline samples $\mathrm{P} 1$ & Arbequina & Producers & 0.11 \\
\hline P2 & Picual & Producers & 0.12 \\
\hline C1 & Arbequina & Retail market & 0.12 \\
\hline $\mathrm{C} 2$ & Hojiblanca & Retail market & 0.13 \\
\hline C3 & Picual & Retail market & 0.14 \\
\hline C6 & $\begin{array}{l}\text { Blend of Hojiblanca, Arbequina, Picual, } \\
\text { Cornicabra }\end{array}$ & Retail market & 0.14 \\
\hline C8 & Arbequina & Retail market & 0.13 \\
\hline \multicolumn{4}{|l|}{ OO samples } \\
\hline O०1 & & $\begin{array}{l}\text { Retail } \\
\text { market }\end{array}$ & 0.28 \\
\hline 002 & & Retail market & 0.34 \\
\hline 003 & & Retail market & 0.41 \\
\hline 004 & & Retail market & 0.41 \\
\hline 005 & & Retail market & 0.41 \\
\hline 006 & & Retail market & 0.48 \\
\hline 007 & & Retail market & 0.50 \\
\hline
\end{tabular}

Table 2. Characteristics of the EVOO samples of set 2 obtained at retail markets analysed by right-angle fluorescence spectroscopy.

\begin{tabular}{lllc}
\hline Sample & Cultivar(s) & Sample & Cultivar \\
\hline $\mathrm{C} 1^{1}$ & Arbequina & $\mathrm{C} 8^{1}$ & Arbequina \\
$\mathrm{C} 2^{1}$ & Hojiblanca & $\mathrm{C} 9$ & Blend \\
$\mathrm{C}^{1}$ & Picual & $\mathrm{C} 10$ & Blend
\end{tabular}




\begin{tabular}{|c|c|c|c|}
\hline C4 & Blend & C11 & Blend \\
\hline C5 & Blend & C12 & Blend \\
\hline $\mathrm{C} 6^{1}$ & $\begin{array}{l}\text { Blend of Hojiblanca, Arbequina, Picual, } \\
\text { Cornicabra }\end{array}$ & C13 & Blend \\
\hline C7 & $\begin{array}{l}\text { Blend of Hojiblanca, Arbequina, Picual, } \\
\text { Cornicabra }\end{array}$ & & \\
\hline
\end{tabular}

3D FF fluorescence spectra were measured using a spectrofluorometer (LS45; PerkinElmer, Waltham, MA, USA) equipped with a xenon lamp source, excitation and emission monochromators and a front-face sample-cell holder. The excitation wavelength ranged from 230 to $646 \mathrm{~nm}$ (step $4 \mathrm{~nm}$ ) and the emission wavelength ranged from 250 to $698.5 \mathrm{~nm}$ (step $0.5 \mathrm{~nm}$ ). Excitation and emission monochromator slit widths were set at $10 \mathrm{~nm}$. Quartz cuvettes $(10 \times 10 \times 45 \mathrm{~mm})$ were filled with the oil samples without prior preparation. For each olive oil sample, a rectangular area was selected that included the region of oxidation products. The excitation band ranged from 274 to $400 \mathrm{~nm}$ and the emission band ranged from 375 to $550 \mathrm{~nm}$. The coefficient of variation (CV) of the signal intensity was computed at every pair $\left(e x_{i}, e m_{j}\right)$ among the samples in both the EVOO group and the 00 group.

For each excitation wavelength, the excitation spectrum was obtained as the sum of the signal along the selected emission wavelengths. The emission spectrum was obtained as the sum of the signal along the selected excitation wavelengths for each emission wavelength. Univariate and multivariate analyses were developed in order to identify the emission wavelengths with potential information related to $\mathrm{K}_{270}$. The univariate approach consisted of computation of the coefficient of determination $\mathrm{R}^{2}$ between the signal intensity at each individual wavelength from 275 to $550 \mathrm{~nm}$ and the analytical $\mathrm{K}_{270}$ values. The multivariate approach computed the b-coefficients of a partial least-square regression (PLSR) for the whole wavelength range. Performance was evaluated through $\mathrm{R}^{2}$ and the biascorrected standard error of prediction $\left(\mathrm{SEP}_{\mathrm{c}}\right)$. RA fluorescence spectra were obtained using a photonic multichannel spectrometer (Hamamatsu, Japan) with detection wavelengths ranging from 196.9 to $958.8 \mathrm{~nm}$ (step $0.75 \mathrm{~nm}$ ). A UV-VIS light source (L10290, Hamamatsu, Japan) was used with a deuterium lamp, with spectral range from 200 to $400 \mathrm{~nm}$. Integration time was set to $1400 \mathrm{~ms}$ and three repetitions per measurement were averaged.

A prototype with a right-angle sample-cell holder was designed and assembled by LPFTAGRALIA. Two optical filters were coupled to the set-up so as to constrain both the excitation and emission wavelength ranges. The optical filter coupled with the fibre coming from the light source allowed a beam with wavelengths below $400 \mathrm{~nm}$. The other optical filter limited the registered beam to wavelengths higher than $400 \mathrm{~nm}$. Measurements were carried out using quartz cuvettes $(10 \times 10 \times 45 \mathrm{~mm})$ directly on the oil samples without prior preparation. The hyperspectral vision system consisted of a push-broom CCD camera (Andor Luca) equipped with a spectrograph Headwall Photonics Hyperspec ${ }^{\mathrm{TM}}$ VNIR $(400-1000 \mathrm{~nm}$, 189 wavelengths, spectral resolution $3.17 \mathrm{~nm}$ ). The illumination was provided by two halogens lamps. The platform moved under the camera and the image was scanned line by line according to the movement (push-broom system). The spatial resolution was $260 \mu \mathrm{m}$. Once the raw images were acquired, the corresponding relative reflectance hypercube was computed, containing the relative reflectance spectrum of each pixel of the image with respect to a reference (mean spectrum of a barium sulfate white reference). Spectra were preprocessed by applying standard normal variate and Savitsky Golay techniques.

\section{RESULTS AND DISCUSSION}

For each olive oil sample of set 1 , a rectangular area was selected that included the region of oxidation products. The excitation band ranged from 274 to $400 \mathrm{~nm}$ and the emission band ranged from 375 to $550 \mathrm{~nm}$. Examples are shown in Figure 1. The CV of the signal intensity was computed at every pair $\left(e x_{i}, e m_{j}\right)$ among the samples in the corresponding group. For EVOO samples, the region with the highest CVs appeared within $e_{i}$ from 274 to $318 \mathrm{~nm}$ and $e m_{j}$ from 380 to $490 \mathrm{~nm}$, i.e., CVs higher than $25 \%$ up to $40 \%$ (red and dark yellow 
in Figure 1). Smaller regions showed CVs around 25\% (light yellow in Figure 1) and a large region showed values around 15\% (light blue in Figure 1).

Higher CVs were found for the OO samples than for EVOO samples. Most of the points within the selected area showed CVs higher than 30\% (Figure 1). Excitation wavelengths around 280, 325 and $345 \mathrm{~nm}$ provided wide emission ranges with the highest CVs, around $35 \%$. According to this observation, sources of information on the oxidation conditions probably arise at least from these three excitation spectral regions. Therefore, a more complete evaluation relies on the registration of the signal resulting from wide bands of excitation wavelengths. When fast monitoring is also required, the optimal solution is to implement a simultaneous excitation at those wavelengths. Another approach was considered by simulating a simultaneous excitation by summing the emission signal along the excitation wavelengths. Two spectral analyses, univariate and multivariate, were developed on the emission spectra in order to identify the emission wavelengths with potential information related to $K_{270} .00$ samples were included so as to increase the range of $K_{270}$ values.
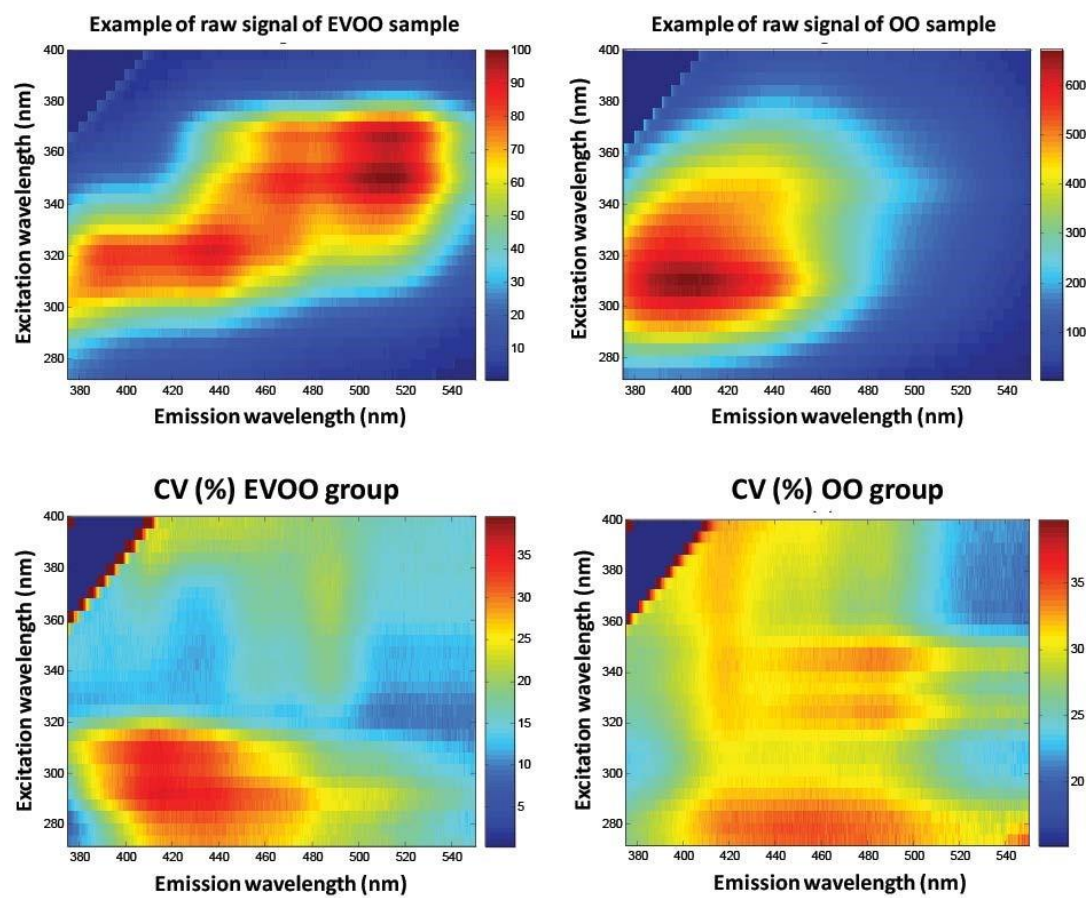

Figure 1. Upper left: example of raw signal from 3D FF spectrum of an EVOO sample. Bottom left: coefficient of variation (CV) (\%) for the group of EVOO samples. Upper right: example of raw signal from 3D FF spectrum of an 00 sample. Bottom right: CV (\%) for the group of 00 samples. Note different colour scales.

For the EVOO group, low $\mathrm{R}^{2}$ values were obtained, below 0.4 , over the whole range. Despite that, two relevant wavelengths can be identified as corresponding to spectral peaks, i.e., around 375 and $443.5 \mathrm{~nm}$, the former with the highest $\mathrm{R}^{2}$ (Figure 2; upper left). As for the OO group, $\mathrm{R}^{2}$ values higher than 0.7 were obtained at $400 \mathrm{~nm}$ and subsequent wavelengths for the whole range. When the whole group was analysed (EVOO and 00), $\mathrm{R}^{2}$ reached values above 0.9 for all wavelengths, with a maximum of 0.98 around $445 \mathrm{~nm}$ (Figure 2; upper left). The high $\mathrm{R}^{2}$ for all emission wavelengths suggests that an intense oxidation produces a general increase in the signal along the whole spectrum, which is related to the overall increase of the $K_{270}$ value. Signal from secondary oxidation products seems to dominate the whole spectra, probably concealing other signals. The multivariate approach identifies a specific emission band ranging from 375 to $450 \mathrm{~nm}$ that provides higher b-coefficients, with a maximum around $410 \mathrm{~nm}$ (Figure 2; upper right). $\mathrm{R}^{2}$ was 0.94 and $\mathrm{SEP}_{\mathrm{c}}$ was 0.0364 using one latent variable. In Figure 2 (bottom left), the intensity of the emission signal at $400 \mathrm{~nm}$ is depicted versus the measured $\mathrm{K}_{270}$ values. In Figure 2 (bottom right), a similar result is obtained when multivariate prediction is illustrated by measured $K_{270}$ values versus predicted $K_{270}$ values for one latent variable. The results obtained are similar to those found in previous studies where 
a single excitation wavelength was used for excitation. Kyriakidis and Skarkalis (2000) used $365 \mathrm{~nm}$ as the excitation wavelength and found an $\mathrm{R}^{2}$ of 0.96 between emission at $445 \mathrm{~nm}$ and $\mathrm{K}_{270}$. Guzmán et al. (2015) found the best performance by applying PLSR with six latent variables for excitation at $380 \mathrm{~nm}$.

Emission spectra ranged from 300 to $800 \mathrm{~nm}$. The root mean square prediction error was 0.08 and the $\mathrm{R}^{2}$ was 0.92 obtained with external validation. However, in the present work, a broad band of excitation wavelengths was studied, with the aim of capturing signal arising from a wider variety of compounds. In view of these results, the signal arising from most of the fluorophores related to the oxidation processes could be obtained with excitation at wavelengths below $400 \mathrm{~nm}$. The emission signals would be related to oxidation by-products and chlorophylls. A wide range of excitation would allow capture of different compounds undergoing production and degradation processes. Therefore, subsequent analyses focused on simultaneous excitation of olive oil samples exposed to indirect light in an RA prototype. At control month, spectra were dominated by signals from chlorophyll at around $670 \mathrm{~nm}$ and the shoulder at $725 \mathrm{~nm}$ (Figure 3). After 2 months, a considerable decrease was observed. Opposite evolution was observed for peaks within the region of oxidation products. Noticeable increases were detected for peaks with local maxima at around 400, 434-437, 464469 and 510-518 nm (Figure 3). Such peaks were in accordance with those found in FF experiments. PC1 loadings in Figure 3 illustrate the variability. Modelling this evolution with regard to official EVOO samples is the next stage that will be faced.
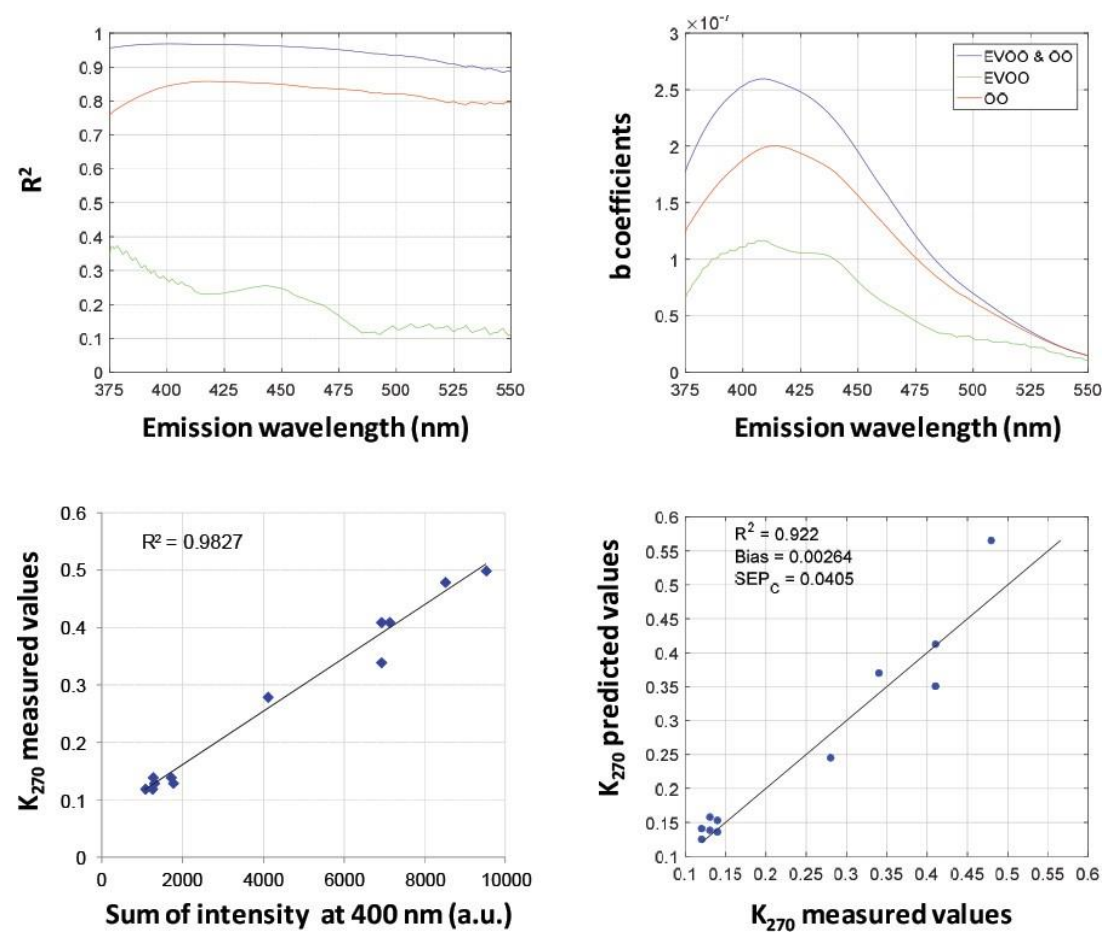

Figure 2. Upper left: coefficient of determination $\mathrm{R}^{2}$ between the signal intensity at each individual wavelength from 275 to $550 \mathrm{~nm}$ and the analytical $\mathrm{K}_{270}$ values. Bottom left: intensity of the sum of emission signal at $400 \mathrm{~nm}$ versus measured $\mathrm{K}_{270}$ values. Upper right: b-coefficients of a PLSR. Bottom right: measured $K_{270}$ values versus predicted $\mathrm{K}_{270}$ values by a PLSR (one latent variable). $\mathrm{SEP}_{\mathrm{c}}$, bias-corrected standard error of prediction.

Spectral patterns were established for each maturity stage according to increasing levels of ripening stage: G1 green olives, G1 'pintonas' olives, G2 purple olives and G3 black olives (Figure 4). Green spectra showed a relevant absorption band at $680 \mathrm{~nm}$, corresponding to high chlorophyll content, and high reflectance at $550 \mathrm{~nm}$, probably related to low anthocyanin content. Average spectra of 'pintonas' olives and purple olives showed similar 
patterns. It seems that these two types of spectra belonged to the same ripening level. These spectra also showed an absorption band at $680 \mathrm{~nm}$, with lower intensity in comparison to the green olive spectra. In addition, the intensity of reflectance at $550 \mathrm{~nm}$ was lower, probably due to higher anthocyanin content. 'Pintonas' olives therefore showed two types of spectra, two different levels of ripening stage within the surface of the fruit. Hyperspectral images are able to sense these differences, in contrast to classical spectroscopy measurements, reflecting non-homogeneities of ripening within the surface of the fruit. Finally, black spectra showed no absorption band at $680 \mathrm{~nm}$ and no reflectance at $550 \mathrm{~nm}$, suggesting total degradation of the chlorophyll and high content of anthocyanins, respectively.
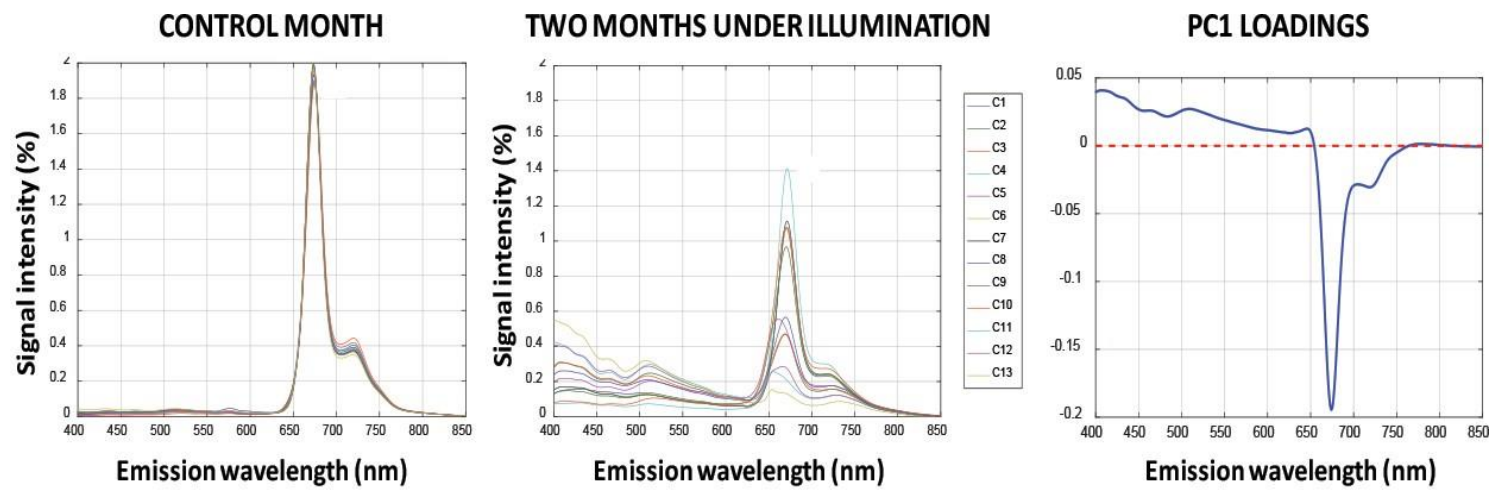

Figure 3. Right-angle fluorescence emission spectra of the commercial EVOO samples. Left: at the control month. Middle: after two months exposed to indirect light. Right: loadings of the first principal component (96.52\% explained variance).
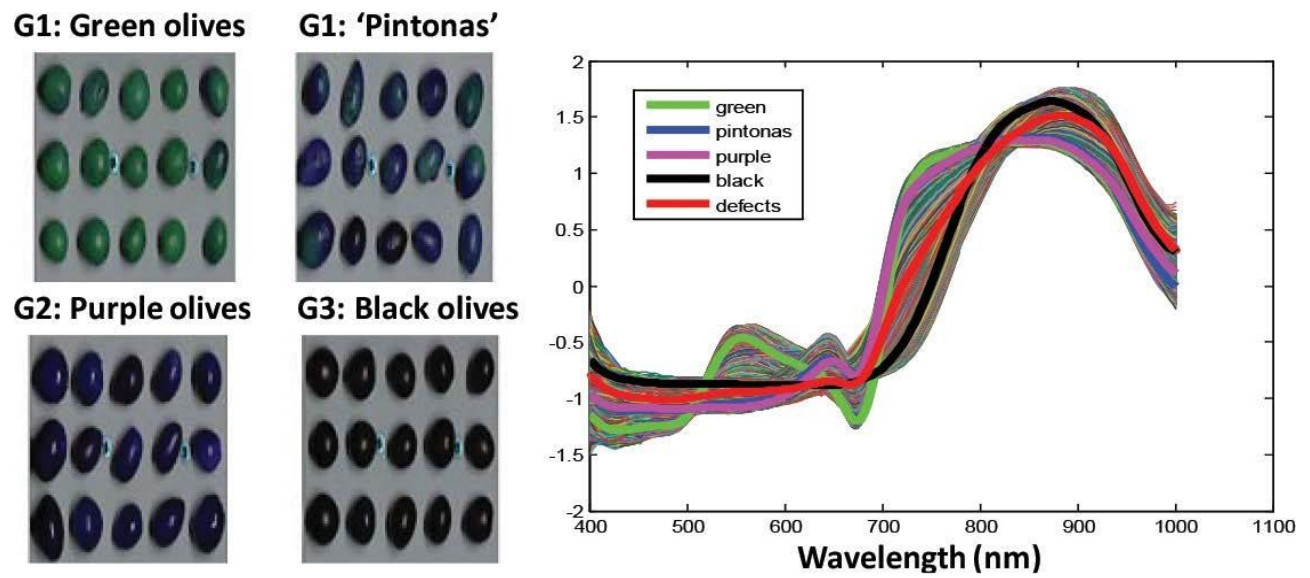

Figure 4. Left: example of images of olives samples in false colour, merging three colour planes: blue, $450 \mathrm{~nm}$; green, 550; red, $680 \mathrm{~nm}$. Right: whole set of pre-processed spectra $(n=13,500)$; average spectrum of each maturity class is plotted.

\section{CONCLUSIONS}

The 3D FF spectra reveal detailed excitation-emission regions. The CV of the signal highlights broad regions related to oxidation processes of olive oil. The emission band from 375 to $550 \mathrm{~nm}$ includes signals produced by an excitation band from 274 to $400 \mathrm{~nm}$ that shows correlation to the characteristic $\mathrm{K}_{240}$, which is identified with advanced oxidation status. Univariate and multivariate approaches provide correlation with this single parameter. Simultaneous excitation with a wide range of wavelengths below $400 \mathrm{~nm}$ in RA experiments is a fast procedure to obtain signals arising from most of the fluorophores related to the complex oxidation processes. The pattern of the spectra acquired using an RA device is in accordance with the pattern of the spectra obtained by integrating the emission signal in FF spectra, showing similar positions of the emission peaks. The RA emission spectral pattern of olive oils exposed to illumination evolves to lower chlorophyll signal and higher signal of oxidation by-products. Ongoing research highlights that hyperspectral images provide spectral patterns of the olives, allowing more precise sorting into categories, which would 
enable the classification into lots of oils with more homogeneous characteristics for subsequent modelling.

\section{ACKNOWLEDGMENTS}

The authors gratefully acknowledge the Centro Tecnológico Agroalimentario de Lugo, Comunidad de Madrid (S2013/ABI-2747, TAVS-CM, Spain), and European Structural Funds for financial support and Juan Ramón Izquierdo from the Laboratorio Arbitral Agroalimentario of MAGRAMA (Spain) for expert advice on olive oil quality, and for providing samples and analytical data. LPF-TAGRALIA is part of the CEI Moncloa Campus of Excellence, UPM-UCM.

\section{Literature cited}

Guzmán, E., Baeten, V., Pierna, J.A., and García-Mesa, J.A. (2015). Evaluation of the overall quality of olive oil using fluorescence spectroscopy. Food Chem. 173, 927-934 https://doi.org/10.1016/j.foodchem.2014.10.041. PubMed

Kyriakidis, N.B., and Skarkalis, P. (2000). Fluorescence spectra measurement of olive oil and other vegetable oils. J. AOAC Int. 83 (6), 1435-1439. PubMed

Lara, M.A., Lleó, L., Diezma-Iglesias, B., Roger, J.M., and Ruiz-Altisent, M. (2013). Monitoring spinach shelf-life with hyperspectral image through packaging films. J. Food Eng. 119 (2), 353-361 https://doi.org/10.1016/j.jfoodeng.2013.06.005.

Lleó, L., Roger, J.M., Herrero-Langreo, A., Diezma-Iglesias, B., and Barreiro, P. (2011). Comparison of multispectral indexes extracted from hyperspectral images for the assessment of fruit ripening. J. Food Eng. 104 (4), 612-620 https://doi.org/10.1016/j.jfoodeng.2011.01.028.

López-Miranda, J., Pérez-Jiménez, F., Ros, E., De Caterina, R., Badimón, L., Covas, M.I., Escrich, E., Ordovás, J.M., Soriguer, F., Abiá, R., et al. (2010). Olive oil and health: summary of the II international conference on olive oil and health consensus report, Jaén and Córdoba (Spain) 2008. Nutr. Metab. Cardiovasc. Dis. 20 (4), 284-294 https://doi.org/10.1016/j.numecd.2009.12.007. PubMed

Sikorska, E., Khmelinskii, I., and Sikorski, M. (2012). Analysis of olive oils by fluorescence spectroscopy: methods and applications. In Olive Oil - Constituents, Quality, Health Properties and Bioconversions, D. Boskou, ed. (Rijeka, Croatia: InTech), p.63-88. 